DIVISION OF THE HUMANITIES AND SOCIAL SCIENCES

CALIFORNIA INSTITUTE OF TECHNOLOGY

PASADENA, CALIFORNIA 91125

MULTI-UTILITARIANISM IN TWO-AGENT QUASILINEAR SOCIAL CHOICE

Christopher P. Chambers

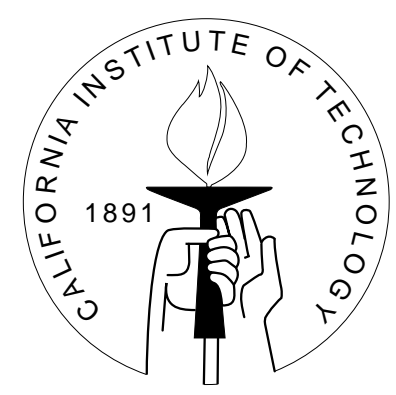

SOCIAL SCIENCE WORKING PAPER 1177

September 2003 


\title{
Multi-Utilitarianism in Two-Agent Quasilinear Social Choice
}

\author{
Christopher P. Chambers
}

\begin{abstract}
We introduce a new class of rules for resolving quasilinear social choice problems. These rules extend those of Green [7]. We call such rules multi-utilitarian rules. Each multi-utilitarian rule is associated with a probability measure over the set of weighted utilitarian rules, and is derived as the expectation of this probability. These rules are characterized by the axioms efficiency, translation invariance, monotonicity, continuity, and additivity. By adding recursive invariance, we obtain a class of asymmetric rules generalizing those Green characterizes. A multi-utilitarian rule satisfying strong monotonicity has an associated probability measure with full support.
\end{abstract}

JEL classification numbers: D63, D70, D71

Key words: social choice, quasilinear bargaining, recursive invariance 


\title{
Multi-Utilitarianism in Two-Agent Quasilinear Social Choice*
}

\author{
Christopher P. Chambers
}

\section{Introduction}

Building on the foundational work of Green $[6,7]$, this paper studies a social choice model where agents can make transfers among themselves. Imagine two agents who must decide on some social alternative. There is an infinitely divisible good, and agents have preferences which are quasilinear over the social alternative and good. The question is which alternative should be chosen, and which transfers should be recommended? We take a normative approach to this problem.

We imagine that randomization over social alternatives is permitted, and that all agents are expected utility maximizers. Moreover, we assume that agents are riskneutral in the infinitely divisible good. Under these assumptions, we may uniquely (up to translation) represent each agent's preference by a utility function which is additively separable in the infinitely divisible good, taking the form $U(p, x)=u(p)+x$.

We do not work with the underlying space of alternatives. Instead, the primitive of the model is the utility possibility set that agents can achieve without making transfers. Thus, the theory is "welfarist," at least in terms of the social alternatives. Any two scenarios which induce the same utility possibility set before transfers are identified. This utility possibility set is referred to as a "problem." For any given problem, a rule specifies a pair of utilities for the agents. We assume this pair of utilities is achievable through transfers.

We discuss properties that rules for solving such problems should satisfy. Minimally, we require that a rule select efficient utility pairs. However; we ask that a rule satisfies several other properties. One such requirement is tied to the underlying utility representation. The utility representation derived for any given preference relation is almost

\footnotetext{
*Assistant Professor of Economics, Division of the Humanities and Social Sciences, 228-77, California Institute of Technology, Pasadena, CA 91125. Email: chambers@hss.caltech.edu. Phone: (626) 3953559. I would like to thank Jerry Green and William Thomson for comments and suggestions. All errors are my own.
} 
unique. However, by adding a constant to a given utility representation, we obtain a new utility representation for a preference which is equally as valid as the first. We will require that a rule is robust to utility specification. Translation invariance states that the addition of a constant vector to a problem (equivalent to adding a constant to each agent's utility function) should induce an equivalent addition of this vector to its solution.

Suppose that a problem is altered, so that alternatives are added which "favor" agent 1, and some alternatives which "favor" agent 2 are removed. How should the solution respond to such a change? As the scenario becomes more favorable toward agent 1, a natural requirement is that the solution should not make agent 1 worse off. This is the axiom of monotonicity.

We further require that the solution to a problem should vary continuously with the problem itself. A rule is continuous if it is continuous with respect to the Hausdorff topology appropriately defined for this model.

Our last axiom states that given two problems, if we know the solution recommended by the rule for each problem, then we can compute the solution for the Minkowski sum of the two problems as the sum of the solutions of the original problems. This axiom is called additivity.

Green [7] investigates the implications of all of the axioms we have discussed, in addition to two other axioms. One of his other axioms is a basic symmetry condition. His other axiom, recursive invariance, is motivated as follows. Suppose a utility pair is selected by a rule for a given problem. Suppose this utility pair is added to the problem, resulting in a modified problem. The rule applied to the modified problem should again select this utility pair. Green characterizes the family of all rules satisfying his axioms. Our result is more general than his, but the rules we characterize share several important characteristics with the rules he characterizes.

Our main result is a characterization of the family of rules satisfying the axioms efficiency, translation invariance, monotonicity, continuity, and additivity. To understand how these rules work, we first discuss the concept of weighted utilitarianism. A weighted utilitarian rule is a rule in which each agent is assigned a nonnegative weight; at least one of which is positive. The agents' weights are not the same. A natural social welfare function over utility space is that which computes the weighted sum of the agents' utilities. A weighted utilitarian rule then works as follows: find the utility pair lying in the problem which induces the maximal social utility. When transfers are possible, this pair is not necessarily efficient. However, there is a remedy for this inefficiency. There exists a unique efficient utility pair (i.e. after transfers) whose social utility is the same as the original utility pair. The weighted utilitarian rule selects this efficient utility pair.

The class of rules satisfying our five axioms is convex. This leads us to a natural conjecture. Suppose we have given a probability distribution over the weighted utilitarian rules. For any given problem, we can compute the expected solution according to this 
distribution. This expected solution is itself a utility pair; thus, we can naturally identify a rule with the probability distribution. Such a rule will be called a "multi-utilitarian rule." Our main contribution is to show that the multi-utilitarian rules are the only rules satisfying the five properties.

Another result that we discuss concerns a weakening of additivity. Suppose that we require that the solution for the "average" of two problems is the average of the solutions. This condition is called mixture linearity, and it plays a fundamental role in the work of Myerson [8]. Mixture linearity is a requirement that precludes a rule from depending on when certain decisions are made. Suppose that a fair coin is tossed to decide which of two problems is to be faced. Mixture linearity requires that the ex-ante expected payoffs to agents do not depend on whether or not the rule is applied before or after the coin toss. We characterize the class of rules satisfying efficiency, translation invariance, monotonicity, continuity, and mixture linearity. These rules are multi-utilitarian rules in which an additional exogenous transfer is made between the agents.

Green's rules are multi-utilitarian rules which feature a probability measure placing positive probability on exactly two weighted utilitarian rules. The two weighted utilitarian rules are symmetric of each other, and have a probability of one-half. We characterize all the multi-utilitarian rules satisfying recursive invariance. We do not require symmetry. A multi-utilitarian rule satisfying recursive invariance places probability on at most two weighted utilitarian rules-one of which favors agent 1, and the other of which favors agent 2 . The weighted utilitarian rules need not be symmetric of each other, and they need not be given equal probability. We call such a rule a "bi-utilitarian rule." It is obvious that by adding symmetry, we obtain Green's rules.

In order to establish that our generalization is useful, we discuss the axiom of strong monotonicity. This axiom states that as a problem becomes more favorable toward agent 1 , then the solution should become more favorable toward agent 1 . None of the multi-utilitarian rules satisfying recursive invariance satisfy strong monotonicity. The class of all multi-utilitarian rules satisfying strong monotonicity is characterized as the set of multi-utilitarian rules whose associated probability measure has full support-what we call a "full multi-utilitarian rule."

Section 2 introduces the formal model. Section 3 includes the main results and proofs. Section 4 concludes.

\section{The model}

\subsection{Preliminaries}

Let $N \equiv\{1,2\}$ be a set of agents. Say that a subset $\mathbf{B} \subset \mathbb{R}^{\mathbf{2}}$ is bounded above if there exists some $x \in \mathbb{R}^{2}$ such that $B \subset\left\{y: y_{1} \leq x_{1}\right.$ and $\left.y_{2} \leq x_{2}\right\}$. A problem is a 


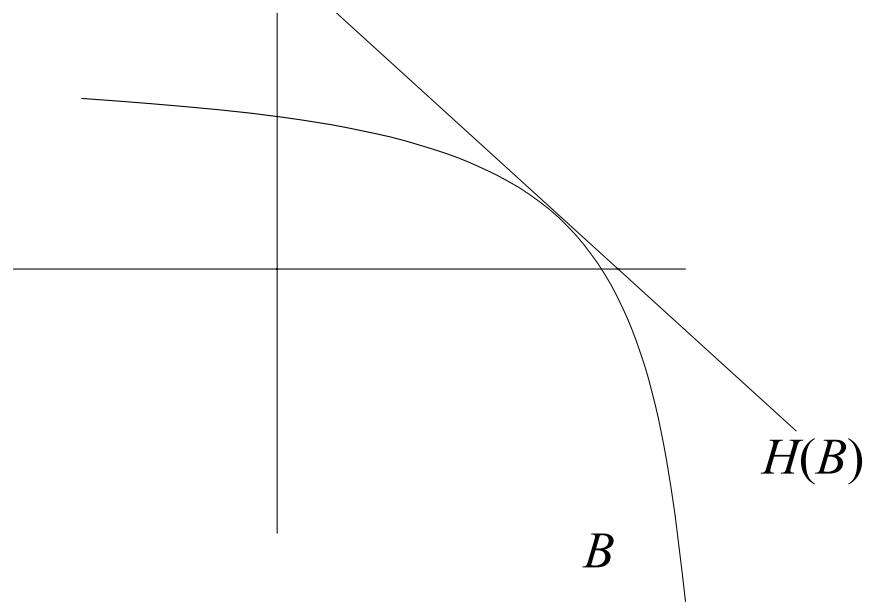

Figure 1: A problem

nonempty subset of $\mathbb{R}^{2}$ which is closed, convex, comprehensive, and bounded above. By $\mathcal{B}$, we mean the set of all problems.

Let $\bar{x}: \mathcal{B} \rightarrow \mathbb{R}$ be defined as $\bar{x}(B) \equiv \max _{x \in B} x_{1}+x_{2}$. We say $x$ is a solution to a problem $B$ if $x_{1}+x_{2} \leq \bar{x}(B)$. Let $H$ be a function defined on the set of problems which maps to the set of hyperplanes of $\mathbb{R}^{2}$. Specifically, let $H(B)$ be defined as $H(B) \equiv$ $\left\{x \in \mathbb{R}^{2}: x_{1}+x_{2}=\bar{x}(B)\right\}$. Thus, $H(B)$ is the set of efficient points that the agents can achieve by making transfers. Figure 1 illustrates a typical problem.

A rule is a function $f: \mathcal{B} \rightarrow \mathbb{R}^{2}$ such that for all $B \in \mathcal{B}, f(B)$ is a solution for $B$. We could conceivably generalize the class of rules to be multi-valued, but for our purposes, single-valued rules will suffice.

\section{$2.2 \quad$ Properties of rules}

We discuss several normative properties that rules may satisfy. The first is the standard concept of efficiency.

Efficiency: For all $B \in \mathcal{B}, f(B) \in H(B)$.

Our next property is to be interpreted as robustness of the rule to the underlying utility specification. Formally, any two problems $B, B^{\prime} \in \mathcal{B}$ such that $B^{\prime}=B+x$ for some $x \in \mathbb{R}^{2}$ can be viewed as arising from the same underlying preferences. Hence, a rule should recommend the same social alternative and transfers in the new problem as in the old problem. But the utility value induced by this solution for the new problem is simply the old utility value, translated by $x$. 


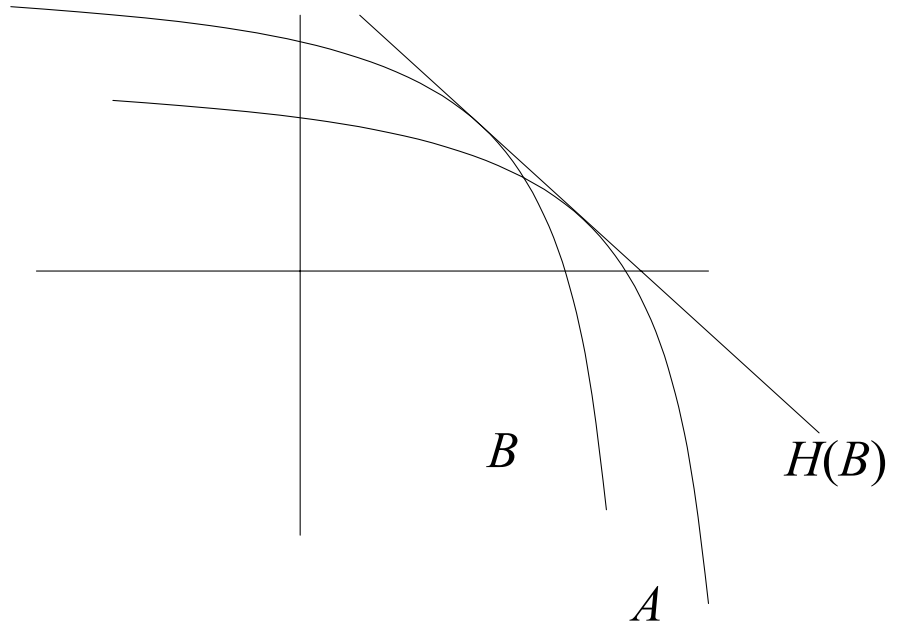

Figure 2: The problem $A$ dominates the problem $B$ for agent 1

Translation invariance: For all $B \in \mathcal{B}$ and all $x \in \mathbb{R}^{2}, f(B+x)=f(B)+x$.

Our next property states that for problems which are the convex and comprehensive hull of singletons, the rule should select that singleton. This axiom is extremely weak and should be interpreted as saying that when there is a single feasible action, this action should be chosen and transfers should not be made.

Formally, $\mathcal{K}$ is a mapping which takes each set into its convex, comprehensive hull.

Selection of singletons: Let $x \in \mathbb{R}^{2}$. Then $f(\mathcal{K}(\{x\}))=x$.

Suppose that we are given two problems $B, B^{\prime} \in \mathcal{B}$. Say that $\mathbf{B}^{\prime}$ dominates $\mathbf{B}$ for agent 1 if $H(B)=H\left(B^{\prime}\right)$ and the following two conditions are satisfied:

$$
\begin{aligned}
& B \cap\left\{x \in \mathbb{R}_{+}: x_{1} \geq \sup \left\{x_{1}: x \in B^{\prime} \cap H\left(B^{\prime}\right)\right\}\right\} \\
\subset \quad & B^{\prime} \cap\left\{x \in \mathbb{R}_{+}: x_{1} \geq \sup \left\{x_{1}: x \in B^{\prime} \cap H\left(B^{\prime}\right)\right\}\right\}
\end{aligned}
$$

and

$$
\begin{aligned}
& B^{\prime} \cap\left\{x \in \mathbb{R}_{+}: x_{2} \geq \sup \left\{x_{2}: x \in B \cap H(B)\right\}\right\} \\
\subset \quad & B \cap\left\{x \in \mathbb{R}_{+}: x_{2} \geq \sup \left\{x_{2}: x \in B \cap H(B)\right\}\right\} .
\end{aligned}
$$

A problem $B^{\prime}$ dominates $B$ for agent 1 if $B^{\prime}$ gives agent 1 "better possibilities" than $B$. We could also define a notion of $B^{\prime}$ dominating $B$ for agent 2 ; the definition is symmetric. Figure 2 illustrates a scenario in which $A$ dominates the set $B$ for agent 1 .

We formulate this condition of domination so that we may discuss a simple monotonicity condition. Thus, imagine $B^{\prime}$ dominates $B$ for agent 1 ; then it is reasonable to require that agent 1 should benefit from this domination. 
Monotonicity: Let $B, B^{\prime} \in \mathcal{B}$ and suppose that $B^{\prime}$ dominates $B$ for agent 1 . Then $f_{1}(B) \leq f_{1}\left(B^{\prime}\right)$.

Monotonicity could also be described using the language of set domination for agent 2; such variants are equivalent under efficiency. Green [7] introduces monotonicity; although his version is weaker.

The next property states that if two problems are "close," then their solutions should be "close." In order to define this, we first define the Hausdorff extended metric on the space $\mathcal{C}$ of closed subsets of $\mathbb{R}^{2}{ }^{1}$ Let $d: \mathbb{R}^{2} \times \mathbb{R}^{2}$ be the Euclidean metric. Define the distance $d^{*}: \mathbb{R}^{2} \times \mathcal{C} \rightarrow \mathbb{R}_{+}$as

$$
d^{*}(x, B) \equiv \inf _{y \in B} d(x, y)
$$

Finally, the Hausdorff extended metric, $d_{\text {Haus }}: \mathcal{C} \times \mathcal{C} \rightarrow \mathbb{R}_{+} \cup\{\infty\}$, is defined as

$$
d_{\text {Haus }}\left(B, B^{\prime}\right) \equiv \max \left\{\sup _{x \in B^{\prime}} d^{*}(x, B), \sup _{x \in B} d^{*}\left(x, B^{\prime}\right)\right\} .
$$

We verify that $d_{\text {Haus }}$ is a metric when restricted to $\mathcal{B} .^{2}$

Proposition 1: The function $d_{\text {Haus }}$ is a metric when restricted to $\mathcal{B}$.

Proof: By Lemma 3.57 of Aliprantis and Border, we know that $d_{\text {Haus }}$ is an extended metric. Thus, we only need establish that for all $B, B^{\prime} \in \mathcal{B}, d_{\text {Haus }}\left(B, B^{\prime}\right)<\infty$. Let $B, B^{\prime} \in \mathcal{B}$. Let $y(B) \equiv\left(\sup _{x \in B} x_{1}, \sup _{x \in B} x_{2}\right)$ and $y\left(B^{\prime}\right) \equiv\left(\sup _{x \in B^{\prime}} x_{1}, \sup _{x \in B^{\prime}} x_{2}\right)$. We claim that $B \cap B^{\prime} \neq \varnothing$. Thus, let $x_{B} \in B$ and let $x_{B^{\prime}} \in B^{\prime}$. Then $x_{B} \wedge x_{B^{\prime}} \in B \cap B^{\prime}$, as $x_{B} \wedge x_{B^{\prime}} \leq x_{B}, x_{B^{\prime}}$, and by comprehensivity of $B$ and $B^{\prime}{ }^{3}$

Thus, let $x^{*} \in B \cap B^{\prime}$. For all $x \leq x^{*}, x \in B \cap B^{\prime}$, and hence $d^{*}(x, B)=0$ and $d^{*}\left(x, B^{\prime}\right)=0$.

We claim that there exists $c_{1}>0$ such that for all $x \in B$ such that $x_{1} \geq x_{1}^{*}$ and $x_{2} \leq x_{2}^{*}, d\left(x, B^{\prime}\right) \leq c_{1}$. Thus, let $x \in B$ satisfy the hypotheses. Set $c_{1} \equiv y_{1}(B)-x_{1}^{*}>$ 0 . Then $x_{1} \leq y_{1}(B)$. Moreover, $\left(x_{1}^{*}, x_{2}\right) \in B^{\prime}$ by comprehensivity of $B^{\prime}$, so that $d\left(\left(x_{1}, x_{2}\right),\left(x_{1}^{*}, x_{2}\right)\right)=x_{1}-x_{1}^{*} \leq c_{1}$.

A similar argument establishes that there exists $c_{2}$ such that for all $x \in B^{\prime}$ such that $x_{1} \geq x_{1}^{*}$ and $x_{2} \leq x_{2}^{*}, d^{*}(x, B) \leq c_{2}$. Moreover, there exists $c_{3}$ such that for all $x \in B$

\footnotetext{
${ }^{1}$ For $d$ to be an extended metric, the following must be true:

i) For all $B, B^{\prime} \in \mathcal{K}, d\left(B, B^{\prime}\right) \geq 0$ with equality if and only if $B=B^{\prime}$

ii) For all $B, B^{\prime} \in \mathcal{K}, d\left(B, B^{\prime}\right)=d\left(B^{\prime}, B\right)$

iii) For all $A, B, C \in \mathcal{K}, d(A, C) \leq d(A, B)+d(B, C)$.

The function $d$ is a metric if it only takes real values.

${ }^{2}$ We slightly abuse notation by referring to $d_{\text {Haus }}$ on $\mathcal{B}$ as $d_{\text {Haus }}$, when it should really be written $\left.d_{\text {Haus }}\right|_{\mathcal{B}}$.

${ }^{3}$ Here, ' $\wedge$ ' refers to the meet of two elements, or the pointwise infimum.
} 
such that $x_{2} \geq x_{2}^{*}$ and $x_{1} \leq x_{1}^{*}, d^{*}\left(x, B^{\prime}\right) \leq c_{3}$. Finally, there exists $c_{4}$ such that for all $x \in B^{\prime}$ such that $x_{2} \geq x_{2}^{*}$ and $x_{1} \leq x_{1}^{*}, d^{*}(x, B) \leq c_{4}$.

Lastly, $A \equiv\left\{x \in B: x \geq x^{*}\right\}$ and $A^{\prime} \equiv\left\{x \in B^{\prime}: x \geq x^{*}\right\}$ are compact sets; hence, $d_{\text {Haus }}\left(A, A^{\prime}\right)<\infty$. By checking the various regions of $B$ and $B^{\prime}$, it is readily verified that

$$
d_{\text {Haus }}\left(B, B^{\prime}\right) \leq \max \left\{c_{1}, c_{2}, c_{3}, c_{4}, \max _{x \in A} d^{*}\left(x, B^{\prime}\right), \max _{x \in A^{\prime}} d^{*}(x, B)\right\},
$$

which is in turn less than or equal to $\max \left\{c_{1}, c_{2}, c_{3}, c_{4}, \max _{x \in A} d\left(x, A^{\prime}\right), \max _{x \in A^{\prime}} d(x, A)\right\}<$ $\infty$.

The space $\mathcal{B}$ is endowed with the topology generated by $d_{\text {Haus }}$. Our next requirement is that a rule is continuous in this topology, called the Hausdorff topology.

Continuity: The rule $f$ is continuous in the Hausdorff topology.

Continuity is a property which is very restrictive in this model. As a natural example of a rule satisfying all of our axioms except for continuity, let $f$ the rule which selects the midpoint of the optimal efficient point for agent 1 and the optimal efficient point for agent 2 .

Lastly, we discuss additivity. For all $A, B \in \mathcal{B}$, define $A+B \equiv$ $\{x+y: x \in A, y \in B\}{ }^{4} \quad$ Note that $A+B \in \mathcal{B}$. Most authors view additivity as a condition which states that a rule is invariant under the sequencing of when problems are faced.

Additivity: For all $A, B \in \mathcal{B}, f(A+B)=f(A)+f(B)$.

Under very mild conditions, additivity is equivalent to the following weaker condition. We use the additivity condition so that the parallels between our work and Green's work are clear.

Mixture linearity: For all $A, B \in \mathcal{B}, f\left(\frac{A+B}{2}\right)=\frac{f(A)+f(B)}{2}$.

Mixture linearity is the requirement that a rule should be invariant to "timing effects." Suppose that the two problems $A$ and $B$ are faced with equal probabilities. Such a scenario induces a natural utility possibility set; $\frac{A+B}{2}$. Applying the rule at this ex-ante stage results in a solution of $f\left(\frac{A+B}{2}\right)$. Waiting until after the randomization to solve the problem results in an ex-ante expected solution of $\frac{f(A)+f(B)}{2}$. Mixture linearity requires that there is no ex-ante benefit to either agent from either procedure.

\footnotetext{
${ }^{4}$ The operator ' + ' is referred to as the Minkowski sum.
} 
Proposition 2: If a rule $f$ satisfies $f(\mathcal{K}(\{0\}))=0$, then it satisfies additivity if and only if it satisfies mixture linearity.

Proof: Let $f$ be a rule satisfying $f(\mathcal{K}(\{0\}))=0$. Suppose that $f$ satisfies additivity. Let $A, B \in \mathcal{B}$. We will show that $f\left(\frac{A+B}{2}\right)=\frac{f(A)+f(B)}{2}$. By additivity, $f\left(\frac{A+B}{2}\right)+f\left(\frac{A+B}{2}\right)=f(A+B)$. By additivity, $f(A+B)=f(A)+f(B)$. Thus, $f\left(\frac{A+B}{2}\right)=\frac{f(A)+f(B)}{2}$. Conversely, suppose that $f$ satisfies mixture linearity. Let $A, B \in \mathcal{B}$. We will show that $f(A+B)=f(A)+f(B)$. Thus, $f\left(\frac{A+B}{2}\right)=$ $f\left(\frac{(A+B)}{2}+\frac{\mathcal{K}(\{0\})}{2}\right)$. By mixture linearity, $f\left(\frac{(A+B)}{2}+\frac{\mathcal{K}(\{0\})}{2}\right)=\frac{f(A+B)+f(\mathcal{K}(\{0\}))}{2}$. By assumption, $\frac{f(A+B)+f(\mathcal{K}(\{0\}))}{2}=\frac{f(A+B)}{2}$. By mixture linearity, $f\left(\frac{A+B}{2}\right)=\frac{f(A)+f(B)}{2}$. Hence, $\frac{f(A+B)}{2}=\frac{f(A)+f(B)}{2}$, so that $f(A+B)=f(A)+f(B)$.

We establish another connection between our axioms which will be useful for the proof of the main result.

Proposition 3: If a rule satisfies additivity, then it satisfies translation invariance if and only if it satisfies selection of singletons.

Proof: Suppose $f$ is additive, and that it satisfies translation invariance. Let $x \in \mathbb{R}^{2}$. Then, by additivity, $f(2 \mathcal{K}(\{0\}))=2 f(\mathcal{K}(\{0\}))$. But by definition, $2 \mathcal{K}(\{0\})=$ $\mathcal{K}(\{0\})$. Thus, $f(\mathcal{K}(\{0\}))=2 f(\mathcal{K}(\{0\}))$, so that $f(\mathcal{K}(\{0\}))=0$. Thus, $f(\mathcal{K}(\{x\}))=$ $f(\mathcal{K}(\{0\})+x)$. By translation invariance, $f(\mathcal{K}(\{0\})+x)=f(\mathcal{K}(\{0\}))+x$. By the preceding statement, $f(\mathcal{K}(\{0\}))+x=x$, so that $f(\mathcal{K}(\{x\}))=x$.

Next, suppose that $f$ satisfies selection of singletons. Then for all $B \in \mathcal{B}$ and all $x \in$ $\mathbb{R}^{2}, f(B+x)=f(B+\mathcal{K}(\{x\}))$. By additivity, $f(B+\mathcal{K}(\{x\}))=f(B)+f(\mathcal{K}(\{x\}))$. By selection of singletons, $f(\mathcal{K}(\{x\}))=x$. Thus, $f(B+x)=f(B)+x$.

\subsection{Multi-utilitarianism}

We now define the set of rules which will be the focus of our study. Let $\lambda \in[0,1]$. For $\lambda \neq 1 / 2$, define the $\lambda$-utilitarian rule $U^{\lambda}: \mathcal{B} \rightarrow \mathbb{R}^{2}$ as follows: for all $B \in \mathcal{B}$,

$$
U^{\lambda}(B) \equiv\left\{y \in H(B): \lambda y_{1}+(1-\lambda) y_{2}=\sup _{x \in B} \lambda x_{1}+(1-\lambda) x_{2}\right\} .
$$

Call this class of rules the weighted utilitarian rules. Clearly, a rule corresponding to $\lambda=1 / 2$ is not well-defined. In standard normative economics, the weighted utilitarian rules select a feasible alternative which maximizes a weighted sum of agents' utilities. Here, such a rule is generally not efficient. Thus, a weighted utilitarian rule specifies an efficient transfer which gives the same aggregate weighted utility as the maximal aggregate weighted utility which is feasible before transfers. Figure 3 illustrates a typical weighted utilitarian rule, here the point $y \equiv U^{x}(B)$. 


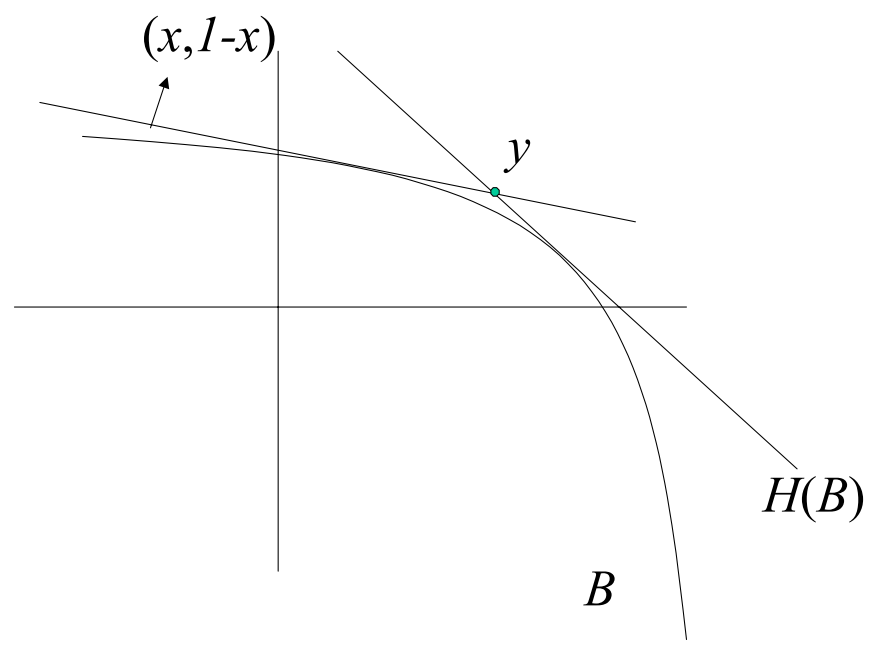

Figure 3: A weighted utilitarian rule

A distinguishing feature of the quasilinear model is that the set of efficient solutions is convex for all problems. This feature allows us to construct many efficient rules out of old rules. Thus, let $\nu$ be a probability measure on the measurable space $([0,1] \backslash\{1 / 2\}, \Sigma)$, where $\Sigma$ are the Borel sets restricted to $[0,1] \backslash\{1 / 2\}$. Define the $\boldsymbol{\nu}$-utilitarian rule $U^{\nu}: \mathcal{B} \rightarrow \mathbb{R}^{2}$ as follows: for all $B \in \mathcal{B}$,

$$
U^{\nu}(B) \equiv \int_{[0,1] \backslash\{1 / 2\}} U^{\lambda}(B) d \nu(\lambda) .
$$

Call this class of rules the multi-utilitarian rules.

\section{Results}

\subsection{The main result}

The main result states that a rule satisfies the five properties if and only if it is a multiutilitarian rule.

Theorem 1: A rule satisfies efficiency, translation invariance, monotonicity, continuity, and additivity if and only if it is a multi-utilitarian rule.

Theorem 1 is tight; we provide the independence of the axioms in the Appendix. 


\subsection{A discussion of proof strategy}

By translation invariance and continuity, we may restrict ourselves to the class of problems whose efficient set consists of points whose aggregate utility is zero and which have a unique efficient utility pair. We can embed any such problem into the space of continuous functions on $[0,1]$. Specifically, each such problem is uniquely determined by the solutions recommended for each of the weighted utilitarian rules. Thus, for a given problem $B, U^{\lambda}(B)$ is a continuous function in $\lambda$ (with the value for $\lambda=1 / 2$ given by $H(B) \cap B$ ). We work on the space of continuous functions which are induced by problems. We define an induced "rule" on this space of functions. It is easily verified that this rule is additive and monotonic (and hence continuous in the sup-norm topology). The rule may be extended to the linear hull of this class of functions, preserving additivity and monotonicity. By using a lattice-theoretic version of the Hahn-Banach theorem, we extend this monotonic functional to the entire space of continuous functions on $[0,1]$ to obtain a monotonic linear functional. Applying the Riesz representation theorem, we conclude that the rule on the space of continuous functions is represented by integration with respect to a measure. Monotonicity guarantees that the measure is positive, and translation invariance guarantees that it assigns measure one to $[0,1]$; hence it is a probability measure. Translating back into the space of problems results in a multi-utilitarian rule on the restricted class of problems. We then show how to extend the characterization on this restricted class to the class of all problems. A similar proof strategy is used in the work of Dekel, Lipman, and Rustichini [4], in the context of choice with unforeseen contingencies.

\subsection{Proof of Theorem 1}

We will not prove that a multi-utilitarian rule satisfies the axioms; this can be easily verified. The opposite direction is proved below.

\section{Proof: Step 1: Establishing the homogeneity of $\mathrm{f}$.}

We claim that for all $B \in \mathcal{B}$ and all $\alpha \geq 0, f(\alpha B)=\alpha f(B)$. Let $\alpha \in \mathbb{Q}$. Then $\alpha=\frac{m}{n}$ for some $m, n \in \mathbb{N}$. Let $x=f\left(\frac{1}{n} B\right)$. Then by additivity, as $B=n\left(\frac{1}{n} B\right)$, $n x=f(B)$. Therefore, $f\left(\frac{1}{n} B\right)=\frac{1}{n} f(B)$. By additivity, $f\left(\frac{m}{n} B\right)=\frac{m}{n} f(B)$, so that $f(\alpha B)=\alpha f(B)$. The result obtains by the density of the rationals and continuity.

Step 2: Embedding rules and problems into the space of continuous functions, and establishing properties on the induced functional.

Define $\mathcal{B}^{*} \subset \mathcal{B}$ to be the class of problems which have a single efficient alternative. Formally, $B \in \mathcal{B}^{*}$ if $H(B) \cap B$ is a singleton. Let $\mathcal{B}^{0} \subset \mathcal{B}^{*}$ so that $B \in \mathcal{B}^{0}$ if $\bar{x}(B)=0$. Most of the work of the proof is done in $\mathcal{B}^{0}$. 
Let $C([0,1])$ be the class of continuous, real-valued functions defined on the unit interval endowed with the sup-norm topology.

Define the function $\sigma: \mathcal{B}^{0} \rightarrow C([0,1])$ by $\sigma(B)(\lambda) \equiv U_{1}^{\lambda}(B)$. It is easily verified that for all $B, \sigma(B)$ is a continuous function on $[0,1]$.

Moreover, $\sigma$ is one-to-one between $\mathcal{B}^{0}$ and $C([0,1])$. It is a simple exercise to verify that for all $B, B^{\prime} \in \mathcal{B}^{0}, \sigma\left(B+B^{\prime}\right)=\sigma(B)+\sigma\left(B^{\prime}\right)$. Further, for all $\alpha \geq 0$ and all $B \in \mathcal{B}^{0}, \sigma(\alpha B)=\alpha \sigma(B)$. (These properties are easily verified, similarly to Rockafellar $[9], 16.4)$.

Moreover, $B^{\prime}$ dominates $B$ for agent 1 if and only if $\sigma\left(B^{\prime}\right) \geq \sigma(B)$. To see this, let $B, B^{\prime} \in \mathcal{B}^{0}$ such that $B^{\prime}$ dominates $B$ for agent 1 . We will show that for all $\lambda \in[0,1 / 2]$, $\sigma\left(B^{\prime}\right)(\lambda) \geq \sigma(B)(\lambda)$. A symmetric argument will establish the proof for $\lambda \in[1 / 2,1]$. Thus, let $\lambda \in[0,1 / 2]$. Let

$$
\begin{aligned}
A^{\prime} & \equiv B^{\prime} \cap\left\{x \in \mathbb{R}_{+}: x_{2} \geq \sup \left\{x_{2}: x \in B \cap H(B)\right\}\right\} \\
A & \equiv B \cap\left\{x \in \mathbb{R}_{+}: x_{2} \geq \sup \left\{x_{2}: x \in B \cap H(B)\right\}\right\} .
\end{aligned}
$$

As $B^{\prime}$ dominates $B$ for agent $1, A^{\prime} \subset A$. Defining $\sup _{x \in \varnothing} f(x)$ as $-\infty$, as $A^{\prime} \subset A$,

$$
\sup _{x \in A^{\prime}} \lambda x_{1}+(1-\lambda) x_{2} \leq \sup _{x \in A} \lambda x_{1}+(1-\lambda) x_{2} .
$$

Let $x^{*} \equiv x \in H(B)$ such that $x_{2}^{*}=\sup \left\{x_{2}: x \in B \cap H(B)\right\}$. We claim that for all $x \in B^{\prime} \backslash A^{\prime}, \lambda x_{1}+(1-\lambda) x_{2} \leq \lambda x_{1}^{*}+(1-\lambda) x_{2}^{*}$. Suppose that this statement is false, so that there exists $x \in B^{\prime} \backslash A^{\prime}$ such that $\lambda x_{1}+(1-\lambda) x_{2}>\lambda x_{1}^{*}+(1-\lambda) x_{2}^{*}$. As $x \notin A^{\prime}$, $x_{2} \leq x_{2}^{*}$. Thus,

$$
\lambda\left(x_{1}-x_{1}^{*}\right)>(1-\lambda)\left(x_{2}^{*}-x_{2}\right),
$$

where the right hand side is nonnegative, as $\lambda<1 / 2$ and $x_{2} \leq x_{2}^{*}$. Conclude that $\lambda\left(x_{1}-x_{1}^{*}\right)>0$; in particular, $\lambda>0$, so that

$$
x_{1}-x_{1}^{*}>\left(\frac{1-\lambda}{\lambda}\right)\left(x_{2}^{*}-x_{2}\right) .
$$

But $\left(\frac{1-\lambda}{\lambda}\right) \geq 1$, so that

$$
x_{1}-x_{1}^{*}>x_{2}^{*}-x_{2}
$$

and

$$
x_{1}+x_{2}>x_{1}^{*}+x_{2}^{*},
$$

contradicting the fact that $H(B)=H\left(B^{\prime}\right)$. It is then clear that

$$
\sup _{x \in B^{\prime}} \lambda x_{1}+(1-\lambda) x_{2} \leq \max \left\{\sup _{x \in A} \lambda x_{1}+(1-\lambda) x_{2}, \lambda x_{1}^{*}+(1-\lambda) x_{2}^{*}\right\},
$$

from which we conclude (using the fact that $A \subset B$ and $x^{*} \in B$ )

$$
\sup _{x \in B^{\prime}} \lambda x_{1}+(1-\lambda) x_{2} \leq \sup _{x \in B} \lambda x_{1}+(1-\lambda) x_{2} .
$$


Next, we claim that for all $\lambda<1 / 2, U_{1}^{\lambda}(B) \leq U_{1}^{\lambda}\left(B^{\prime}\right)$ if and only if $\sup _{x \in B^{\prime}} \lambda x_{1}+$ $(1-\lambda) x_{2} \leq \sup _{x \in B} \lambda x_{1}+(1-\lambda) x_{2}$. To this end, we need only establish that $U_{1}^{\lambda}(B) \leq$ $U_{1}^{\lambda}\left(B^{\prime}\right)$ implies $\sup _{x \in B^{\prime}} \lambda x_{1}+(1-\lambda) x_{2} \leq \sup _{x \in B} \lambda x_{1}+(1-\lambda) x_{2}$ and that $U_{1}^{\lambda}(B)<$ $U_{1}^{\lambda}\left(B^{\prime}\right)$ implies $\sup _{x \in B^{\prime}} \lambda x_{1}+(1-\lambda) x_{2}<\sup _{x \in B} \lambda x_{1}+(1-\lambda) x_{2}$. We will show the second statement; the first follows by replacing all strict inequalities by weak inequalities. By definition,

$$
\lambda U_{1}^{\lambda}\left(B^{\prime}\right)+(1-\lambda)\left(-U_{1}^{\lambda}\left(B^{\prime}\right)\right)=\sup _{x \in B^{\prime}} \lambda x_{1}+(1-\lambda) x_{2}
$$

and

$$
\lambda U_{1}^{\lambda}(B)+(1-\lambda)\left(-U_{1}^{\lambda}(B)\right)=\sup _{x \in B} \lambda x_{1}+(1-\lambda) x_{2} .
$$

As $\lambda<1 / 2, \lambda\left(U_{1}^{\lambda}\left(B^{\prime}\right)-U_{1}^{\lambda}(B)\right)<(1-\lambda)\left(U_{1}^{\lambda}\left(B^{\prime}\right)-U_{1}^{\lambda}(B)\right)$. Hence $\lambda U_{1}^{\lambda}\left(B^{\prime}\right)+$ $(1-\lambda)\left(-U_{1}^{\lambda}\left(B^{\prime}\right)\right)<\lambda U_{1}^{\lambda}(B)+(1-\lambda)\left(-U_{1}^{\lambda}(B)\right)$.

Thus, for all $\lambda<1 / 2$, we conclude that $\sigma(B)(\lambda) \leq \sigma\left(B^{\prime}\right)(\lambda)$. The argument for $\lambda>1 / 2$ is symmetric. Lastly, the argument for $\lambda=1 / 2$ follows from the fact that for all $\lambda \neq 1 / 2, \sigma(B)(\lambda) \leq \sigma\left(B^{\prime}\right)(\lambda)$, and since each of $\sigma(B)$ and $\sigma\left(B^{\prime}\right)$ are continuous functions.

Next, we show that if $\sigma(B) \leq \sigma\left(B^{\prime}\right)$, then $B^{\prime}$ dominates $B$ for agent 1 . So, let $\lambda<$ $1 / 2$. By the argument above, if $\sigma(B) \leq \sigma\left(B^{\prime}\right)$, we conclude $\sup _{x \in B^{\prime}} \lambda x_{1}+(1-\lambda) x_{2} \leq$ $\sup _{x \in B} \lambda x_{1}+(1-\lambda) x_{2}$. Suppose that there exists some $y^{*} \in B^{\prime}$ such that $y_{2}^{*} \geq x_{2}^{*}$ and $y^{*} \in B^{\prime} \backslash B$. We will derive a contradiction. By a version of the Separating Hyperplane Theorem (Corollary 5.59 of Aliprantis and Border [1]), there exists a pair $(\lambda, 1-\lambda) \in \mathbb{R}^{2}$ such that $\lambda y_{1}^{*}+(1-\lambda) y_{2}^{*}>\sup _{x \in B} \lambda x_{1}+(1-\lambda) x_{2}$. We claim that $\lambda \in[0,1 / 2]$. Clearly, $\lambda \in[0,1]$, or else the supremum over $B$ does not exist. So, suppose that $\lambda>1 / 2$. In particular, $\lambda y_{1}^{*}+(1-\lambda) y_{2}^{*}>\lambda x_{1}^{*}+(1-\lambda) x_{2}^{*}$, from which we conclude that $\lambda\left(y_{1}^{*}-x_{1}^{*}\right)>(1-\lambda)\left(x_{2}^{*}-y_{2}^{*}\right)$. As $\lambda>0$, this implies that $y_{1}^{*}-x_{1}^{*}>\left(\frac{1-\lambda}{\lambda}\right)\left(x_{2}^{*}-y_{2}^{*}\right)$. Next, as $y_{2}^{*}>x_{2}^{*}$, and as $\left(\frac{1-\lambda}{\lambda}\right)<1$, we conclude $\left(\frac{1-\lambda}{\lambda}\right)\left(x_{2}^{*}-y_{2}^{*}\right)>x_{2}^{*}-y_{2}^{*}$. Hence $y_{1}^{*}-x_{1}^{*}>x_{2}^{*}-y_{2}^{*}$, or $y_{1}^{*}+y_{2}^{*}>x_{1}^{*}+x_{2}^{*}$, contradicting $H(B)=H\left(B^{\prime}\right)$. Thus, $\lambda \in[0,1 / 2]$. But then $\sup _{x \in B^{\prime}} \lambda x_{1}+(1-\lambda) x_{2} \geq \lambda y_{1}^{*}+(1-\lambda) y_{2}^{*}>\sup _{x \in B} \lambda x_{1}+(1-\lambda) x_{2}$, so that $\sigma\left(B^{\prime}\right)(\lambda)>\sigma(B)(\lambda)$, a contradiction.

Define $T: \sigma\left(\mathcal{B}^{0}\right) \rightarrow \mathbb{R}$ as $T(\sigma(B)) \equiv f_{1}(B)$. The function $T$ is then additive and homogeneous on $\sigma\left(\mathcal{B}^{0}\right)$. As $f$ is monotonic, then by the preceding statements, $T$ is monotonic, and hence continuous in the sup-norm topology.

\section{Step 3: Extending the linear functional to the space of continuous func- tions.}

We extend $T$ to a vector subspace of $C([0,1])$. Thus, let $\mathcal{H} \equiv\left\{g-h: g, h \in \sigma\left(\mathcal{B}^{0}\right)\right\}$. Clearly, $\mathcal{H}$ is now a vector subspace. Define $T^{*}: \mathcal{H} \rightarrow \mathbb{R}$ by $T^{*}(g-h)=T(g)-T(h)$. We claim that $T^{*}$ is well-defined, linear, and continuous. To see that it is well-defined, suppose that $g-h \in \mathcal{H}$ can be written as $g-h=g^{\prime}-h^{\prime}$. Thus, $g+h^{\prime}=g^{\prime}+h$. 
We conclude that $T\left(g+h^{\prime}\right)=T\left(g^{\prime}+h\right)$; moreover, by additivity of $T, T\left(g+h^{\prime}\right)=$ $T(g)+T\left(h^{\prime}\right)$ and $T\left(g^{\prime}+h\right)=T\left(g^{\prime}\right)+T(h)$. Therefore, $T(g)+T\left(h^{\prime}\right)=T\left(g^{\prime}\right)+T(h)$. Hence, $T(g)-T(h)=T\left(g^{\prime}\right)-T\left(h^{\prime}\right)$. Therefore, $T^{*}$ is well-defined. As $T$ is linear, so is $T^{*}$. As $T$ is monotonic, $T^{*}$ is monotonic. To see this, suppose that $g-h \geq 0$. Then $T^{*}(g-h)=T(g)-T(h)$. Since $g \geq h$, and as $T$ is monotonic, $T(g)-T(h) \geq 0$. Hence $T^{*}$ is monotonic, and hence continuous.

We extend $T^{*}$ to all of $C([0,1])$. We can extend $T^{*}$ to all of $C([0,1])$ so that the extension is monotonic (Corollary III.9.12 of Conway [3], using the fact that $1 \in \mathcal{H}$, where $1=\sigma(\mathcal{K}(\{(1,-1)\})))$. We refer to this continuous linear extension as $T^{* *}$.

\section{Step 4: Obtaining the measure representation of the rule for a restricted class of problems.}

By the Riesz representation theorem (for example, see Corollary 13.15 of Aliprantis and Border [1]), there exists a countably additive measure $\nu$ on $([0,1], \Sigma)$ such that

$$
T^{* *}(f) \equiv \int_{[0,1]} f(\lambda) d \nu(\lambda)
$$

Further, $\nu$ is positive if $T^{* *}$ is monotonic.

We claim that for all $c \in \mathbb{R}, T^{* *}(c)=c .^{5} \quad$ It is clear by definition that $\sigma(\mathcal{K}(\{(c,-c)\}))$ is the constant function $c$. Moreover, we know by selection of singletons that $f(\mathcal{K}(\{(c,-c)\}))=(c,-c)$. Thus, by definition of $T, T(c)=c$ and hence $T^{* *}(c)=c$. As for all constant functions $c, T^{* *}(c)=c$, we conclude that $T^{* *}(c)=\nu([0,1]) c=c$, so that $\nu([0,1])=1$.

By definition of $T$, for all $B \in \mathcal{B}^{0}, f(B)=(T(\sigma(B)),-T(\sigma(B)))=$ $\left(\int_{[0,1]} \sigma(B)(\lambda) d \nu(\lambda),-\int_{[0,1]} \sigma(B)(\lambda) d \nu(\lambda)\right)$. Rewriting,

$$
f(B)=\int_{[0,1]}(\sigma(B)(\lambda),-\sigma(B)(\lambda)) d \nu(\lambda) .
$$

For all $\lambda,(\sigma(B)(\lambda),-\sigma(B)(\lambda))=U^{\lambda}(B)$. Thus,

$$
f(B)=\int_{[0,1]} U^{\lambda}(B) d \nu(\lambda) .
$$

We show that this formula holds for all $B \in \mathcal{B}^{*}$. Let $B \in \mathcal{B}^{*}$ and let $x$ satisfy $B+x \in \mathcal{B}^{0}{ }^{6} \quad$ Then $f(B+x)=\int_{[0,1]} U^{\lambda}(B+x) d \nu(\lambda)$. For all $\lambda, U^{\lambda}$ is translation invariant (it can easily be shown to hold for $\lambda=1 / 2$ ), so that $U^{\lambda}(B+x)=U^{\lambda}(B)+x$.

\footnotetext{
${ }^{5}$ We abuse notation in a standard way by identifying a constant function with the value that constant function takes.

${ }^{6}$ For example, let $x=(-\bar{x}(B), 0)$.
} 
Hence $f(B+x)=\int_{[0,1]}\left(U^{\lambda}(B)+x\right) d \nu(\lambda)$. As $\nu([0,1])=1$, the preceding is equal to $\int_{[0,1]} U^{\lambda}(B) d \nu(\lambda)+x$. By translation invariance of $f, f(B+x)=f(B)+x$. Hence, $f(B)+x=\int_{[0,1]} U^{\lambda}(B) d \nu(\lambda)+x$, so that $f(B)=\int_{[0,1]} U^{\lambda}(B) d \nu(\lambda)$.

Step 5: Verifying that $1 / 2$ has measure zero, and completing the characterization.

We extend the representation to all of $\mathcal{B}$. First, we establish that $\nu(\{1 / 2\})=$ 0 . Let $\left\{B_{n}\right\},\left\{B_{n}^{\prime}\right\} \subset \mathcal{B}$ be the following sequences of problems: for all $n, B_{n} \equiv$ $\mathcal{K}\left(\left\{\left(1-\frac{1}{n},-1\right),(0,0)\right\}\right)$ and $B_{n}^{\prime} \equiv \mathcal{K}\left(\left\{(1,-1),\left(0,-\frac{1}{n}\right)\right\}\right)$. Then, note that each of $B_{n}$ and $B_{n}^{\prime}$ converge to $\mathcal{K}(\{(1,-1),(0,0)\})$ in the Hausdorff topology. Thus, by continuity, $\lim _{n \rightarrow \infty} f\left(B_{n}\right)=\lim _{n \rightarrow \infty} f\left(B_{n}^{\prime}\right)$. In particular, we can identify each $B_{n}$ and $B_{n}^{\prime}$ with its induced continuous function, $\sigma\left(B_{n}\right)$ and $\sigma\left(B_{n}^{\prime}\right)$. It is simple to verify that the sequence $\sigma\left(B_{n}\right)$ converges pointwise to

$$
F(\lambda) \equiv\left\{\begin{array}{l}
0 \text { for } \lambda \leq 1 / 2 \\
1 \text { for } \lambda>1 / 2
\end{array}\right.
$$

and that the sequence $\sigma\left(B_{n}^{\prime}\right)$ converges pointwise to

$$
F^{\prime}(\lambda) \equiv\left\{\begin{array}{l}
0 \text { for } \lambda<1 / 2 \\
1 \text { for } \lambda \geq 1 / 2
\end{array}\right.
$$

In particular,

$$
\lim _{n \rightarrow \infty} \int_{[0,1]} \sigma\left(B_{n}\right)(\lambda) d \nu(\lambda)=\lim _{n \rightarrow \infty} \int_{[0,1]} \sigma\left(B_{n}^{\prime}\right)(\lambda) d \nu(\lambda) .
$$

Each of $\sigma\left(B_{n}\right)$ and $\sigma\left(B_{n}^{\prime}\right)$ are bounded sequences; hence, we may apply the Lebesgue dominated convergence theorem (for example, see Theorem 11.20 of Aliprantis and Border [1]). We conclude

$$
\int_{[0,1]} F(\lambda) d \nu(\lambda)=\int_{[0,1]} F^{\prime}(\lambda) d \nu(\lambda) .
$$

Moreover, $\int_{[0,1]} F(\lambda) d \nu(\lambda)=\nu((1 / 2,1])$ and $\int_{[0,1]} F^{\prime}(\lambda) d \nu(\lambda)=\nu([1 / 2,1])$. Thus $\nu((1 / 2,1])=\nu([1 / 2,1])$, or $\nu(\{1 / 2\})=0$.

As the set $\mathcal{B}^{*}$ is dense in $\mathcal{B}$ in the Hausdorff topology, we establish that for all $B \in \mathcal{B}$,

$$
f(B)=\int_{[0,1]} U^{\lambda}(B) d \nu(\lambda)
$$

independently of how $U^{1 / 2}$ is defined. We may thus write

$$
f(B)=\int_{[0,1] \backslash\{1 / 2\}} U^{\lambda}(B) d \nu(\lambda) .
$$




\subsection{A characterization on the basis of mixture linearity}

We establish a characterization of a class of rules based on mixture linearity. Fix a multi-utilitarian rule, $U^{\nu}$. Fix some exogenous transfer from agent 2 to agent 1 , say, $c$. For any problem $B$, such a rule recommends whatever is recommended by $U^{\nu}$, plus the transfer from agent 2 to agent 1.

Corollary 1: A rule $f$ satisfies efficiency, translation invariance, monotonicity, continuity, and mixture linearity if and only if there exist $c \in \mathbb{R}$ and a multi-utilitarian rule $U^{\nu}$ such that for all $B \in \mathcal{B}, f(B)=(c,-c)+U^{\nu}(B)$.

Proof: It is simple to verify that any such rule satisfies the axioms.

Conversely, let $f$ be a rule satisfying the axioms. By efficiency, the sum of the elements of $f(\mathcal{K}(\{0\}))$ is equal to zero. Thus, let $(c,-c) \equiv f(\mathcal{K}(\{0\}))$.

Let $f^{*}$ be the rule defined so that for all $B \in \mathcal{B}, f^{*}(B) \equiv f(B)-(c,-c)$. Then it is trivial to verify that $f^{*}$ satisfies the axioms listed in the hypothesis of the corollary. Moreover, $f^{*}(\mathcal{K}(\{0\}))=0$. Thus, by Proposition 2, we may conclude that $f^{*}$ is additive. Hence $f^{*}$ is a multi-utilitarian rule, say $U^{\nu}$. Therefore, $f(B) \equiv(c,-c)+U^{\nu}(B)$.

\subsection{On Green's Theorem and recursive invariance}

Green's theorem invokes all of the axioms we discuss (except for a weaker version of monotonicity), in addition to the following. It states that if a solution for a problem is determined by a rule, then adding this solution to the utility possibilities set should not change the solution selected by the rule.

Recursive invariance: For all $B \in \mathcal{B}, f(\mathcal{K}(B \cup\{f(B)\}))=f(B)$.

Green attributes this axiom to Chun [2], although Chun never actually uses it in any characterization. Together with a basic symmetry condition, Green characterizes a oneparameter subset of the multi-utilitarian rules, which for lack of better terminology, we call class $\mathcal{G}$. Members of $\mathcal{G}$ are described as follows. Fix a parameter $\lambda \in[0,1 / 2)$. Let $\nu^{\lambda}$ be the probability measure such that $\nu^{\lambda}(\{\lambda\})=\nu^{\lambda}(\{1-\lambda\})=1 / 2$. The elements of $\mathcal{G}$ are the multi-utilitarian rules corresponding to such probability measures.

We will show how to derive a result related to Green's from ours as a corollary, without using the symmetry axiom. The proof of Green's main result relies on a beautiful functional equations argument. Here, our argument is primarily measure-theoretic.

Define a generalization of Green's rules as follows. Say a multi-utilitarian rule $f$ is a bi-utilitarian rule if its associated probability measure $\nu$ has a support of at most two elements, one of which lies above $1 / 2$ and the other of which lies below $1 / 2$ (recall that the 
support of a probability measure is the intersection of all closed sets having probability one). ${ }^{7}$ A bi-utilitarian rule need not place equal probability on each of the two weighted utilitarian rules with which it is associated. In fact, it might place positive probability on only one weighted utilitarian rule; such a rule is itself a weighted utilitarian rule. It is clear that requiring symmetry will force a bi-utilitarian rule to be an element of $\mathcal{G}$.

Corollary 2: A rule satisfies efficiency, translation invariance, monotonicity, continuity, additivity, and recursive invariance if and only if it is a bi-utilitarian rule.

Proof: To show that a bi-utilitarian rule satisfies the axioms is simple. Conversely, suppose $f$ is a rule satisfying the six axioms. By means of contradiction, suppose that $f$ is not a bi-utilitarian rule. By Theorem $1, f$ is a multi-utilitarian rule. Let $\nu$ be the probability measure associated with $f$. As $f$ is not a bi-utilitarian rule, we may assume without loss of generality that the support of $\nu$ contains more than one element which is greater than $1 / 2$.

We will show that the support of $\nu$ contains at most one point greater than $1 / 2$. To this end, suppose by means of contradiction that it contains at least two. Let $\lambda^{*}>1 / 2$ be an element in the support which is strictly less than the supremal element.

We now construct a problem which is the intersection of the hyperplanes in the directions $\left(\lambda^{*}, 1-\lambda^{*}\right),(1,0)$, and $(0,1)$. Thus, let $B \equiv\left\{x \in \mathbb{R}^{2}: \lambda_{1}^{*} x_{1}+\left(1-\lambda_{1}\right) x_{2}^{*} \leq 0\right\} \cap$ $\left\{x \in \mathbb{R}^{2}: x_{1} \leq 1\right\} \cap\left\{x \in \mathbb{R}^{2}: x_{2} \leq 0\right\}$. Then for all $\lambda \leq \lambda^{*}$, (including $\lambda<1 / 2$ ), $U^{\lambda}(B)=0$, and for all $\lambda>\lambda^{*}, U_{1}^{\lambda}(B)>0$. As the support of $\nu$ contains points which are greater than $\lambda^{*}$ (as $\lambda^{*}$ was less than the supremal element), we conclude that $U_{1}^{\nu}(B)>0$. In fact, there exists some $\lambda^{\prime}>\lambda^{*}$ such that for all $\lambda \in\left(1 / 2, \lambda^{\prime}\right)$, $U_{1}^{\lambda}\left(\mathcal{K}\left(B \cup\left\{U^{\nu}(B)\right\}\right)\right)=U_{1}^{\nu}(B)>U_{1}^{\lambda}(B)$. For all other $\lambda, U^{\lambda}\left(\mathcal{K}\left(B \cup\left\{U^{\nu}(B)\right\}\right)\right)=$ $U^{\lambda}(B)$. The set $\left(1 / 2, \lambda^{\prime}\right)$ has positive measure according to $\nu$, so that this implies $U_{1}^{\nu}\left(\mathcal{K}\left(B \cup\left\{U^{\nu}(B)\right\}\right)\right)>U_{1}^{\nu}(B)$. This is a contradiction to recursive invariance.

\subsection{On strongly monotonic multi-utilitarian rules}

A natural question is whether or not there are interesting multi-utilitarian rules which do not belong to $\mathcal{G}$. The following axiom, which is a strengthening of monotonicity, is violated by all members of $\mathcal{G}$.

Strong monotonicity: Let $B, B^{\prime} \in \mathcal{B}$ and suppose that $B^{\prime}$ dominates $B$ for agent 1 and that $B \neq B^{\prime}$. Then $f_{1}(B)<f_{1}\left(B^{\prime}\right)$.

\footnotetext{
${ }^{7} \mathrm{An}$ important feature of the definition of a bi-utilitarian rule is that there can be at most one weighted utilitarian rule favoring agent 1 and at most one favoring agent 2 . In other words, it cannot put support on two weighted utilitarian rules, each of which favor agent 1 . This is not indicated in the simple terminology "bi-utilitarian."
} 
Multi-utilitarian rules satisfying strong monotonicity exist, and in fact a characterization of this family is possible. We demonstrate that a multi-utilitarian rule satisfies strong monotonicity if and only if the associated probability measure has a support of $[0,1] \backslash\{1 / 2\}$ in the relative topology on $[0,1] \backslash\{1 / 2\}$. Say a rule is a full multiutilitarian rule if it is a multi-utilitarian rule whose associated probability measure has full support.

Corollary 3: A rule satisfies efficiency, translation invariance, strong monotonicity, continuity, and additivity if and only if it is a full multi-utilitarian rule.

Proof: It is simple to show that a full multi-utilitarian rule satisfies strong monotonicity.

We prove the other direction. Let $f$ be a rule satisfying the five axioms listed in the theorem. Strong monotonicity implies monotonicity, so by Theorem $1, f$ is a multiutilitarian rule. Let $\nu$ be the probability measure associated with $f$. It is enough to show that for all open intervals $\left(\lambda_{1}, \lambda_{2}\right)$ with $\lambda_{1}<\lambda_{2}$, which do not include $1 / 2, \nu\left(\left(\lambda_{1}, \lambda_{2}\right)\right)>0$. Let $\left(\lambda_{1}, \lambda_{2}\right)$ be such an interval, and without loss of generality, suppose that $\lambda_{1}>1 / 2$.

We construct two problems, $B^{\prime}$ and $B$. Let

$$
\begin{aligned}
B^{\prime} \equiv & \left\{x \in \mathbb{R}^{2}: \lambda_{1} x_{1}+\left(1-\lambda_{1}\right) x_{2} \leq 0\right\} \cap\left\{x \in \mathbb{R}^{2}: \lambda_{2} x_{1}+\left(1-\lambda_{2}\right) x_{2} \leq 0\right\} \\
& \cap\left\{x \in \mathbb{R}^{2}: x_{1} \leq 1\right\} \cap\left\{x \in \mathbb{R}^{2}: x_{2} \leq 1\right\} .
\end{aligned}
$$

Let $B \equiv \mathcal{K}\left(\left\{\left(1, \frac{\lambda_{1}-1}{\lambda_{1}}\right),\left(\frac{\lambda_{2}-1}{\lambda_{2}}, 1\right)\right\}\right)$. The problem $B^{\prime}$ is the convex, comprehensive hull of $B$ with the origin. The important point (which is also easily verified) is that $B^{\prime}$ dominates $B$ for agent 1 and for all $\lambda \notin\left(\lambda_{1}, \lambda_{2}\right), U^{\lambda}(B)=U^{\lambda}\left(B^{\prime}\right)$. By strong monotonicity, $f_{1}\left(B^{\prime}\right)>f_{1}(B)$. Thus, by definition of $f$,

$$
\int_{[0,1] \backslash\{1 / 2\}} U_{1}^{\lambda}\left(B^{\prime}\right) d \nu(\lambda)>\int_{[0,1] \backslash\{1 / 2\}} U_{1}^{\lambda}(B) d \nu(\lambda) .
$$

Rewriting,

$$
\int_{[0,1] \backslash\{1 / 2\}} U_{1}^{\lambda}\left(B^{\prime}\right)-U_{1}^{\lambda}(B) d \nu(\lambda)>0,
$$

and as for all $\lambda \notin\left(\lambda_{1}, \lambda_{2}\right), U^{\lambda}(B)=U^{\lambda}\left(B^{\prime}\right)$, conclude

$$
\int_{\left(\lambda_{1}, \lambda_{2}\right)} U_{1}^{\lambda}\left(B^{\prime}\right)-U_{1}^{\lambda}(B) d \nu(\lambda)>0
$$

establishing that $\nu\left(\left(\lambda_{1}, \lambda_{2}\right)\right)>0$. Thus $f$ is a full multi-utilitarian rule. 


\section{Conclusion}

A last point that bears mentioning: Green discusses a strengthening of continuity which involves the "bounded convergence topology." A sequence $\left\{B_{n}\right\}$ converges to $B$ in the bounded convergence topology if and only if for all compact sets $K, K \cap B_{n}$ converges to $K \cap B$. Continuity with respect to the bounded convergence topology is stronger than continuity with respect to the Hausdorff topology; for example, the sequence $\mathcal{K}(\{(0,0),(1,-n)\})$ converges to $\mathcal{K}(\{(0,0)\})$ as $n \rightarrow \infty$ in the bounded convergence topology, but not in the Hausdorff topology. Strengthening continuity in Theorem 1 in this sense results in the additional implication that $\nu(\{0\})=\nu(\{1\})=0$.

Extending the families of rules characterized in this work to environments involving many agents is the subject of ongoing research.

\section{Appendix: On the independence of the axioms in Theorem 1}

In this Appendix, for each axiom used in the characterization provided in Theorem 1, we provide an example of a rule (or family of rules) which violates this axiom, yet satisfies the remaining axioms of Theorem 1.

Example 1: A rule that satisfies translation invariance, monotonicity, continuity, and additivity but not efficiency. Let $f(B) \equiv U^{0}(B) \wedge U^{1}(B)$. As is stated in the text, monotonicity has a parallel statement for agent 2 (which is equivalent to the original statement of monotonicity under the efficiency axiom). The rule $f$ satisfies the alternative version of monotonicity.

Example 2: A rule that satisfies efficiency, monotonicity, continuity, and additivity but not translation invariance. Let $f(B)$ be the point of equal coordinates on $H(B)$. Thus, $x=f(B)$ if and only if $x_{1}=x_{2}$ and $x \in H(B)$.

Example 3: A rule that satisfies efficiency, translation invariance, continuity, and additivity but not monotonicity. This class of rules can be characterized (by strengthening continuity to Lipschitz continuity), and we will call them the generalized multi-utilitarian rules. Formally, let $\nu$ be a countably additive signed measure of bounded variation on $([0,1] \backslash\{1 / 2\}, \Sigma)$ satisfying $\nu([0,1] \backslash\{1 / 2\})=1$. Define $U^{\nu}$ such that for all $B \in \mathcal{B}$,

$$
U^{\nu}(B) \equiv \int_{[0,1] \backslash\{1 / 2\}} U^{\lambda}(B) d \nu(\lambda) .
$$


Example 4: A rule that satisfies efficiency, translation invariance, monotonicity, additivity but not continuity. An example of this type was already provided in the text. For another example, give agent 1 his supremal utility in the efficient set:

$$
f(B) \equiv\left\{x \in \overline{H(B)}: x_{1} \geq y_{1} \text { for all } y \in H(B)\right\}
$$

Example 5: A rule that satisfies efficiency, translation invariance, monotonicity, and continuity but not additivity. This is perhaps the easiest example to think of. A simple example, inspired by the decision theory literature (see Gilboa and Schmeidler [5]) is the following. Let $\Pi$ be a convex and weak* compact set of Borel probability measures over $[0,1] \backslash\{1,2\}$. Define

$$
f(B) \equiv\left(\min _{p \in \Pi} \int_{[0,1] \backslash\{1 / 2\}} U_{1}^{\lambda}(B) d p(\lambda), \bar{x}(B)-\min _{p \in \Pi} \int_{[0,1] \backslash\{1 / 2\}} U_{1}^{\lambda}(B) d p(\lambda)\right) .
$$

Then this rule satisfies all of the axioms but additivity.

\section{References}

[1] C.D. Aliprantis and K. Border, "Infinite Dimensional Analysis: a Hitchhiker's Guide," 2nd edition, Springer-Verlag, New York, 1999.

[2] Y. Chun, Agreement, separability, and other axioms for quasi-linear social choice problems, Seoul Journal of Economics 2 (1989), 225-244.

[3] J.B. Conway, "A Course in Functional Analysis," second edition, Springer-Verlag, 1990 .

[4] E. Dekel, B.L. Lipman, and A. Rustichini, Representing preferences with a unique subjective state space, Econometrica 69 (2001), 891-934.

[5] I. Gilboa and D. Schmeidler, Maxmin expected utility with nonunique prior, Journal of Mathematical Economics 18 (1989), 141-153.

[6] J. Green, "A Theory of Bargaining with Monetary Transfers," Discussion Paper Number 966, Harvard Institute of Economic Research, 1983.

[7] J. Green, "Compensatory Transfers in Two-Player Decision Problems," mimeo, Harvard University, 2003.

[8] R. Myerson, Utilitarianism, egalitarianism, and the timing effect in social choice problems, Econometrica 49 883-897, (1981).

[9] R.T. Rockafellar, "Convex Analysis," Princeton University Press, Princeton, NJ, 1970 . 